\title{
Composición, estructura y diversidad florística del bosque seco en el Valle de Agalta, Honduras
}

\author{
Composition, structure, and floristic diversity of the dry forest of Agalta \\ Valley, Honduras
}

\author{
Lilian Ferrufino-Acosta', Saby Y. Cruz', Thelma Mejía-Ordóñez', Fabiola Rodríguez²,3, Dorian Escoto', \\ Elia Sarmiento' y Jeffery L. Larkin ${ }^{2,3}$
}

\begin{abstract}
Universidad Nacional Autónoma de Honduras (UNAH), Facultad de Ciencias. Escuela de Biología. Tegucigalpa, Francisco Morazán, Honduras.

\author{
Universidad Indiana de Pennsylvania. Instituto de \\ Investigación. Indiana, PA, Estados Unidos. \\ 3 American Bird Conservancy. The Plains. Virginia, \\ Estados Unidos
}

\section{RESUMEN}

Los bosques secos se consideran uno de los ecosistemas más amenazados en el trópico por las actividades antrópicas que han fragmentado y reducido el hábitat. Este estudio documentó la composición florística del bosque seco del Valle de Agalta en Honduras, con énfasis en los sitios donde habita el colibrí esmeralda (Amazilia luciae), un ave endémica y en peligro de extinción. Se colectaron datos de la estructura y composición vegetal de 265 parcelas en 35 sitios. Se identificaron 316 especies distribuidas en 76 familias y 222 géneros, que incluyen $79 \%$ eudicotiledóneas, $15 \%$ monocotiledóneas, $8 \%$ monilófitas y 1\% gimnospermas. Las distribuciones vertical y horizontal indican que el bosque está dominado por individuos jóvenes. Las asociaciones vegetales más dominantes, según el índice de valor de importancia, fueron Acacia picachensis (Fabaceae), Eugenia hondurensis (Myrtaceae) y Lysiloma acapulcense (Fabaceae). Se concluye que el Valle de Agalta aún posee bosque seco, que representa un hábitat único y es el refugio de muchas especies nativas, raras y endémicas, enlistándose algunas especies de alta prioridad de conservación.

PALABRAS CLAVE: Amazilia luciae; asociaciones vegetales; biodiversidad; bosque seco tropical; estructura vegetal; Fabaceae.

\section{ABSTRACT}

Dry forests are considered one of the most threatened ecosystems in the tropics due to anthropogenic activities that cause fragmentation and habitat loss. Structural and plant composition data were collected from 265 plots in 35 sites, resulting in the identification of 316 species distributed in 76 families and 222 genera, including $79 \%$ eudicot, 15\% monocot, 8\% monilophytes, and 1\% gymnosperms. The vertical and horizontal distribution indicated that the forest is dominated by young individuals. Acacia picachensis (Fabaceae), Eugenia hondurensis (Myrtaceae), and Lysiloma acapulcense (Fabaceae) were identified as the most dominant plant species based on the importance value index. The remaining Agalta Valley's dry forest represents a unique habitat and is the refuge of many native, rare, and endemic species, including some considered high conservation priority.

KEYWORDS: Amarilia luciae; plant assemblages; biodiversity; tropical dry forest; vegetation structure; Fabaceae.

\section{INTRODUCCIÓN}

Los bosques secos están entre los ecosistemas tropicales más amenazados y menos estudiados, en comparación con los bosques húmedos (Janzen, 1988; Whitmore, 1998; Espinosa, de la Cruz, Luzuriaga y Escudero, 2012). Estos ecosistemas presentan un alto endemismo y diversidad en plantas. Sin embargo, las especies vegetales se encuentran en peligro de extinción debido principalmente al impacto causado por actividades antrópicas como la ganadería, la agricultura, la tala, el urbanismo, incendios forestales, altas temperaturas, la 
lixiviación de nutrientes en los suelos desde los agrosistemas, entre otros (Wright y Müller-Landau, 2006; Miles et al., 2006; Pennington, Lewis y Ratter, 2006; Finegan y Bouroncle, 2007; Ruíz y Fandiño, 2009). Esta situación ha causado que Latinoamérica y el Caribe cuente con menos del 10\% del área original de bosque seco (Banda et al., 2016).

Existen áreas de bosque seco en Mesoamérica que se consideran intactas y con un manejo sostenible con relación a la agricultura, como es el caso del bosque de la costa del Estado de Oaxaca en México (Barrance, Schreckenberg y Gordon, 2009). En Honduras, por el contrario, los bosques secos al sur del país han sido casi completamente convertidos en agroecosistemas dominados por el cultivo de granos básicos, con remanentes de bosque secundario fragmentado (Barrance et al., 2009). La distribución de especies en estos remanentes de bosque seco de especial interés para la conservación de Honduras no es uniforme; los ecosistemas semiáridos del Aguan, Agalta, Comayagua y Tegucigalpa poseen un mayor número de especies amenazadas y endémicas en comparación con los ecosistemas de bosques deciduos del Pacífico, Olancho y el Valle de Sula (Midence, 2016). No cabe duda de que existe escasa información sobre este ecosistema en el país siendo el principal motivo la falta de interés por el gobierno, lo que permite percibir que aún falta por describir muchas especies endémicas y amenazadas que habitan en estos remanentes (Secretaría de Energía, Recursos Naturales, Ambiente y Minas, 2014).

Se han realizado estudios florísticos en el bosque seco tropical (BST) en Honduras a escala local (Duery Salek, 2001; Cardona Caravantes y Oramas Wenholz, 2002; Gutiérrez y Linares Escalante, 2002; Menéndez Posada y Melara Estrada, 2002; House, 2004; Pérez-Laniado, 2004; Garcés-López, 2004; Illescas-Burneo, 2005; Carbajal Vásquez, 2010; Mora, Espinal, López y Quezada, 2015; Ferrufino-Acosta, Díaz, Benítez-Nassar, Atao, GarcíaChaclan, López y Pilz, 2018a; Ferrufino-Acosta, Sandoval y Oyuela, 2018b). Este estudio pretende contribuir al conocimiento florístico de los bosques secos de Honduras a través del estudio de diversidad de plantas leñosas y herbáceas del BST del Valle de Agalta.

\section{OBJETIVOS}

Los objetivos específicos de esta investigación fueron: 1) describir la composición florística del bosque seco en el Valle de Agalta; 2) determinar la composición y estructura del bosque seco del Valle de Agalta a través del uso de distintas medidas estructurales; 3) realizar un inventario de plantas de las especies de importancia para la conservación según La Unión Internacional para la Conservación de la Naturaleza (UICN) y la Convención sobre el Comercio Internacional de Especies Amenazadas de Fauna y Flora Silvestre (CITES) para la protección del hábitat del Colibrí Esmeralda en Valle de Agalta (Amazilia luciae).

\section{MATERIALES Y MÉTODOS}

\section{Área de estudio}

El Valle de Agalta, ubicado en el departamento de Olancho

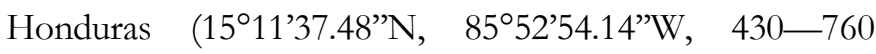
msnm; Fig. 1), es un área que posee las siguientes zonas de vida: bosque seco tropical (principalmente), bosque húmedo subtropical, bosque húmedo montano bajo y bosque muy húmedo montano bajo (Holdridge, 1962; NASA Land Processes Distributed Active Archive Center [LP DAAC ], 2001). De acuerdo con la información más reciente de cobertura de suelo para Honduras (Instituto Nacional de Conservación y Desarrollo Forestal, Áreas Protegidas y Vida Silvestre [ICF], 2014), en el Valle de Agalta los tipos de usos del suelo y cobertura abarcan la agricultura tecnificada, árboles dispersos, bosque seco hoja ancha, bosque mixto, pastos y/o cultivos, vegetación seca secundaria y vegetación seca húmeda. La precipitación media anual es $1140 \mathrm{~mm}$, la temperatura media máxima anual $31.1^{\circ} \mathrm{C}$ y la mínima anual $19.46{ }^{\circ} \mathrm{C}$, comprendiendo febrero, marzo y abril los meses más secos (Centro Internacional de Agricultura Tropical [CIAT], 1999a). Según la clasificación del Centro Internacional de Agricultura Tropical [CIAT], (1999b) los tipos de suelo presentes en el Valle son: Ustropepts típicos y suelos asociados a partir de aluvión viejo en terrazas (ITUa1), Haplustolls líticos y suelos asociados a partir de caliza en laderas (MUHi-1) y Haplustolls líticos y suelos asociados a partir de caliza en montañas (MUHi-2). 


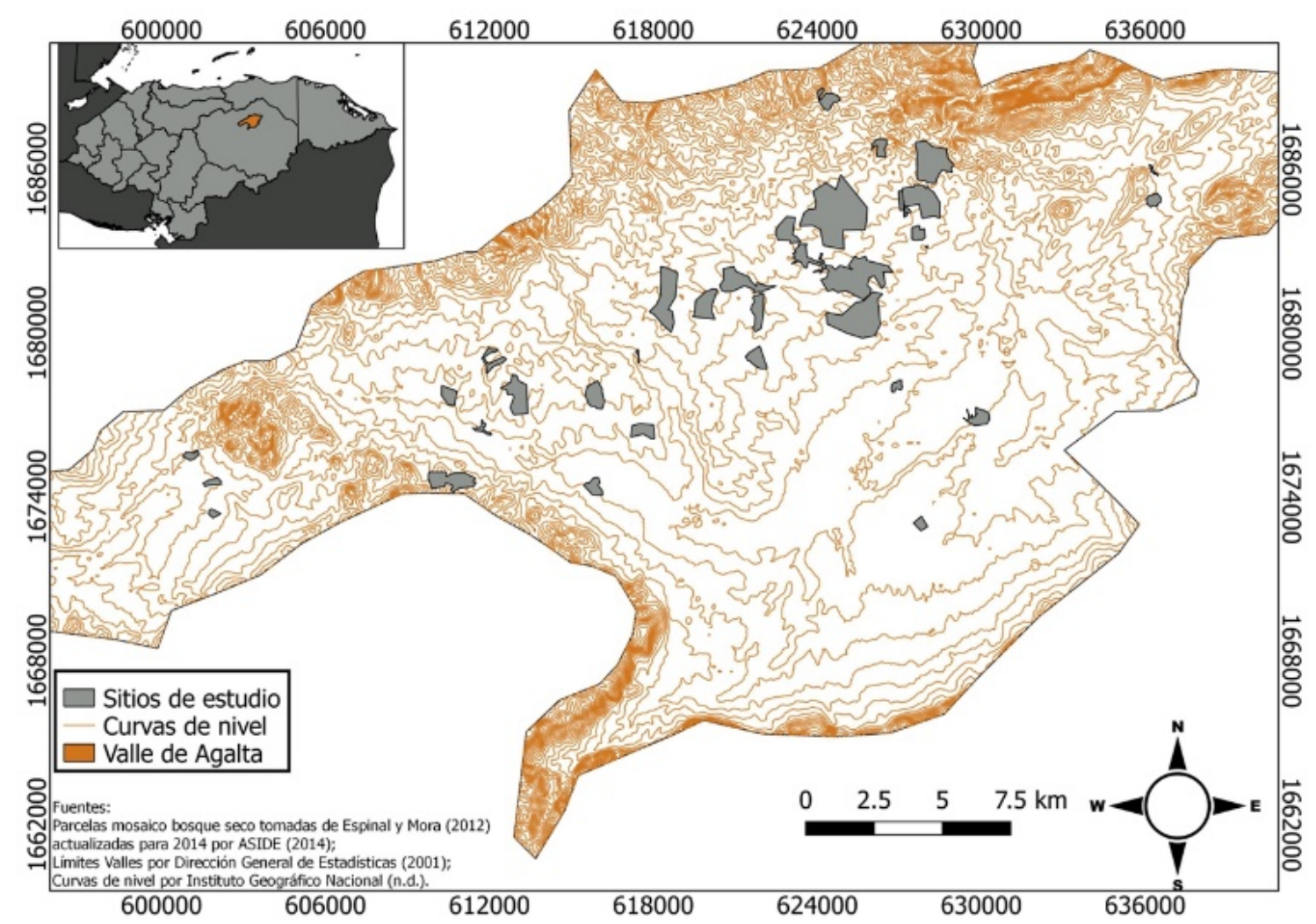

FIGURA 1. Delimitación y localización del Valle de Agalta, en el Departamento de Olancho, Honduras. Sistema de coordenadas de referencia: WGS 8416 N UTM.

\section{Muestreo}

Para el muestreo de vegetación se seleccionaron 35 sitios siguiendo un esquema estratificado que comprendió bosque seco compuestos de: matorral seco, bosque dominado por dosel, bosque de galería y áreas designadas para el uso de ganado. Los sitios fueron seleccionados basado en dos criterios: 1) presencia al menos de un fragmento de bosque seco (matorral o dominado por dosel) como parte del mosaico y 2) contar con acceso por parte de los propietarios. Dentro de los sitios de estudio, se muestrearon 265 puntos (parcelas) generados de manera aleatoria.

\section{Muestreo composición florística y estructura vegetal}

El muestreo se realizó de enero a julio de 2015. Las muestras se marcaron con el mismo código del área de la parcela circular. Se colectaron 480 muestras botánicas, y estas fueron identificadas y depositadas en el Herbario Cyril
Hardy Nelson Sutherland (TEFH) de la Universidad Nacional Autónoma de Honduras. Para determinar la estructura y composición florística se estableció una "parcela circular anidada" (James y Shugart, 1970) que comprendió: una parcela principal con un radio de $12.6 \mathrm{~m}$ (área igual a 0.05 ha) para el muestreo de árboles, conteniendo una segunda sub-parcela circular de $5 \mathrm{~m}$ de radio para muestrear el estrato arbustivo, y finalmente una tercera de $1 \mathrm{~m}$ de radio para muestrear el estrato herbáceo. Siguiendo los métodos propuestos por Bibby, Burgess, Hill y Mustoe (1992), Martin, Paine, Conway, Hochachka, Allen y Jenkins (1997), Nassar, Rodríguez, Sánchez-Azofeifa, Garvin y Quesada (2008) y García-Villacorta (2009), en cada parcela se midió el DAP (diámetro a la altura del pecho, 1.30 $\mathrm{m}$ ) de cada tallo de árbol $\geq 3 \mathrm{~cm}$ de diámetro y se estimó la altura de árboles (m), altura de arbustos (m), altura de cactáceas (m), cobertura de dosel (\%) a nivel de parcela y porcentajes de cobertura de suelo para las categorías de herbáceas, hojarasca, suelo desnudo u otros (\%). 


\section{Análisis de datos}

Para describir la estructura ecológica, se obtuvo un resumen de datos con las siguientes mediciones y se calculó la media \pm error estándar para cada parámetro: promedio del DAP, altura promedio de árboles $(\mathrm{m})$ y arbustos $(\mathrm{m})$, área basal/parcela $\left(\mathrm{m}^{2} / \mathrm{ha}\right)$, densidad de árboles (tallos $\geq 3 \mathrm{~cm}$ $\mathrm{DAP} / \mathrm{ha}$ ), densidad tallos $<3 \mathrm{~cm} \mathrm{DAP}$ (número de tallos de árboles por hectárea), densidad arbustos (número de tallos de arbustos/ha), densidad cactáceas (número de cactus por hectárea), altura promedio de cactáceas (m), cobertura dosel (\%), coeficiente de variación (\%) para cada uno de los parámetros ecológicos, altura de árboles, altura de arbustos, altura de cactáceas, densidad de arbustos, densidad de cactáceas y densidad de árboles. Se calculó los siguientes índices para evaluar la estructura ecológica: índice de valor de importancia (IVI) (Curtis y McIntosh 1951), índice de complejidad de Holdridge (Holdridge, 1967), índice de diversidad Shannon-Wiener (Hill, 1973). Se transformaron los datos de abundancias para especies de plantas leñosas $\geq 3 \mathrm{~cm}$ DAP en cada parcela utilizando la distancia de Hellinger (Rao, 1995).

Además, se calculó el coeficiente de similitud, basada en la distancia euclidiana (Orloci, 1967) y posteriormente un análisis jerárquico de aglomeración con el método de Ward de varianza mínima (Ward 1963). La transformación de las abundancias y la distancia euclideana se obtuvo en el paquete "vegan" (Oksanen et al., 2015) y el algoritmo de aglomeración de Ward se calculó como especifican Murtagh y Legendre (2014) con el paquete "stats" de R (R Core Team, 2016). El análisis de aglomeración y los datos asociados a este análisis se condujo utilizando una muestra de 261 puntos de los 265 muestreados, debido a que cuatro sitios no presentaron plantas leñosas $\geq 3 \mathrm{~cm}$ DAP. Se incluyó en el análisis 82 especies de plantas leñosas y se excluyó las especies que ocurrieron únicamente en un sitio de acuerdo a lo propuesto por Austin y Greig-Smith (1968) y Burgman y Thompson (1982).

Los datos de abundancia absoluta para cada especie leñosa de $\geq 3 \mathrm{~cm}$ DAP por parcela, fueron utilizados para elaborar una curva de acumulación de especies. Se estimó la riqueza total con el estimador no-paramétrico Jackknife de primer orden el cual se considera contribuye a los cálculos de riqueza con buen desempeño al disminuir el sesgo y mejorar la precisión (Chiarucci, 2001; Gotelli y Colwell 2011; Walther y Moore 2005) usando el paquete "vegan" (Oksanen et al., 2015) en la plataforma de R (R Core Team, 2016).

\section{Resultados}

\section{Composición florística}

Un total de 316 especies de plantas se identificaron, representando 76 familias y 222 géneros (Suplemento 1). Todas las especies registradas son en su mayoría eudicotiledóneas (79\%), seguidas de las monocotiledóneas $(15 \%)$, monilófitas $(5 \%)$ y gimnospermas $(1 \%)$. La mayoría de las especies son nativas y el $4 \%$ son especies introducidas. La mayoría de las especies registradas son arborescentes (arboles 35\% y arbustos 18\%) y herbáceas (47\%). Asimismo, en el Valle se registran especies endémicas siendo: Bakeridesia molinae, Opuntia hondurensis, Eugenia hondurensis, Robinsonella erasmi-sosae y Rondeletia bondurensis.

\section{Estructura ecológica}

La familia Fabaceae fue la más diversa (42 spp.), seguida por Malvaceae (23 spp.) y Euphorbiaceae (16 spp.). Según el IVI, las diez familias más comunes enlistan: Fabaceae, Malvaceae, Euphorbiaceae, Capparaceae, Rubiaceae, Bromeliaceae, Orchidaceae, Asteraceae, Verbenaceae y Convolvulaceae. Así como las diez especies más abundantes fueron: Acacia picachensis, Engenia hondurensis, Casearia nitida, Erythroxylum areolatum, Zanthoxylum fagara, Neomillspanghia paniculata, Triumfetta calderonii, Bursera simaruba, Coccoloba acapulcensis, y Luehea candida (Fig. 2).

El dosel se compone de algunas especies arbóreas más dominantes del bosque, siendo: Acacia pennatula, Lonchocarpus guatemalensis, Luehea candida, Eugenia hondurensis, Coccoloba acapulcensis, Randia aculeata, Zanthoxylum fagara, Casearia aculeata, Sideroxylon capiri, 
Guaiacum sanctum, Quadrella lundellii, Rhus terebinthifolia, Symplocos matudae, entre otras. Las especies que habitan en el sotobosque son: Solanum dasyanthum, Schoepfia schreberi, Bernardia nicaraguensis, Eupatorium crassirameum, Lippia cardiostegia, Waltheria indica, Hechtia guatemalensis, Aechmea bracteata, Opuntia bondurensis, Mimosa tenuiflora entre otras.

En la distribución horizontal de los sitios de muestreo, se registraron 16249 tallos $\geq 3 \mathrm{~cm}$ DAP. El promedio de DAP fue $6.31 \mathrm{~cm} \pm 0.04 \mathrm{~cm}$ (intervalo $=3 \mathrm{~cm}-112 \mathrm{~cm}$ ), con $85 \%$ de los tallos entre $3 \mathrm{~cm}-9 \mathrm{~cm}$. El porcentaje más bajo $(1.87 \%)$ fue registrado para todos los tallos $>20 \mathrm{~cm}$ DAP (Fig. 3a). La distribución vertical presentó un promedio de $6.14 \mathrm{~m} \pm 0.03 \mathrm{~m}$. Se identificaron tres estratos de menor tamaño, árboles menores a $6 \mathrm{~m}$ representaron $51 \%$ de los individuos registrados (Fig. 3b). Se reportaron 113 especies leñosas $>3 \mathrm{~cm}$ DAP que fueron muestreadas sistemáticamente en 261 parcelas. El estimado de riqueza total fue $143.8 \pm 6.8$ (error estándar) y la figura 4 indica que se requirieron más de 100 parcelas de muestreo para registrar más de 100 especies leñosas.

\section{Comunidades vegetales}

Se identificó cinco comunidades florísticas (Fig. 5 y 6). A continuación, se describe cada comunidad observada y los parámetros de estructura vegetal (Tablas 1,2 y 3).

Comunidad 1: Esta comunidad ( $\mathrm{n}=50$ parcelas, Figura $7 \mathrm{a}$ ) se caracterizó por poseer alta abundancia relativa (69.39\%) de Acacia picachensis, seguida de porcentajes más bajos (1\%$4 \%$ ) de otras especies leñosas como Casearia nitida, Bursera simaruba, Pithecellobium dulce, Leucaena leucocephala, Randia aculeata, Erythroxylum areolatum, Neomillspanghia paniculata, Chloroleucon mangense, Coccoloba acapulcensis y Acacia collinsi. Además $A$. picachensis resultó con el mayor IVI para esta comunidad (55.5\%). En esta comunidad se incluyen las mayores alturas de árboles de la distribución vertical del Valle de Agalta.
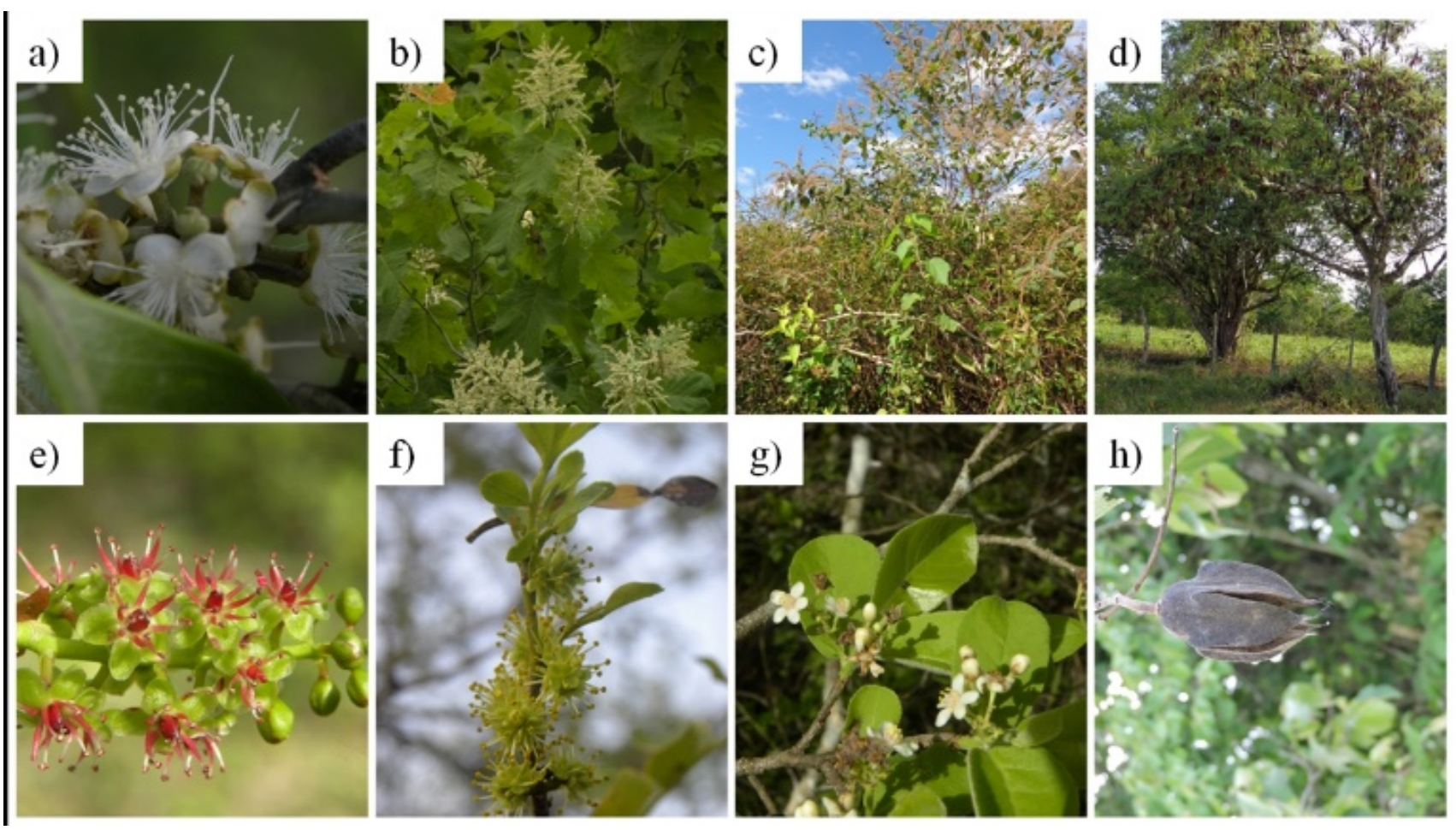

FIGURA 2. Especies más abundantes en el BST del Valle de Agalta, Honduras. a) Eugenia hondurensis; b) Neomillspaughia paniculata; c) Triumfetta calderon; d) Acacia picachensis; e) Coccoloba acapulcensis; f) Casearia nitida, g) Zanthoxylum fagara y h) Luehea candida. 
a)

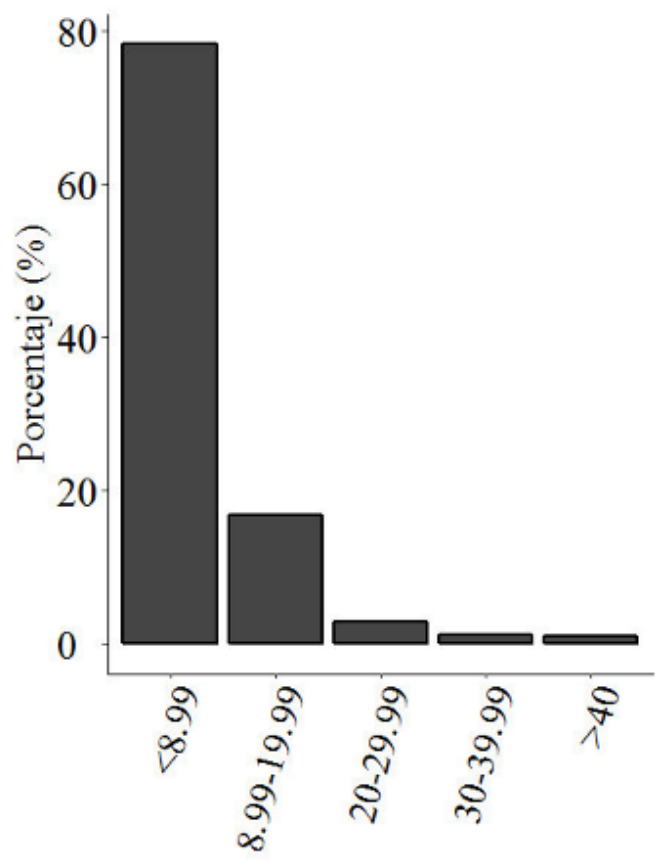

Clases diamétricas DAP $(\mathrm{cm})$ b)

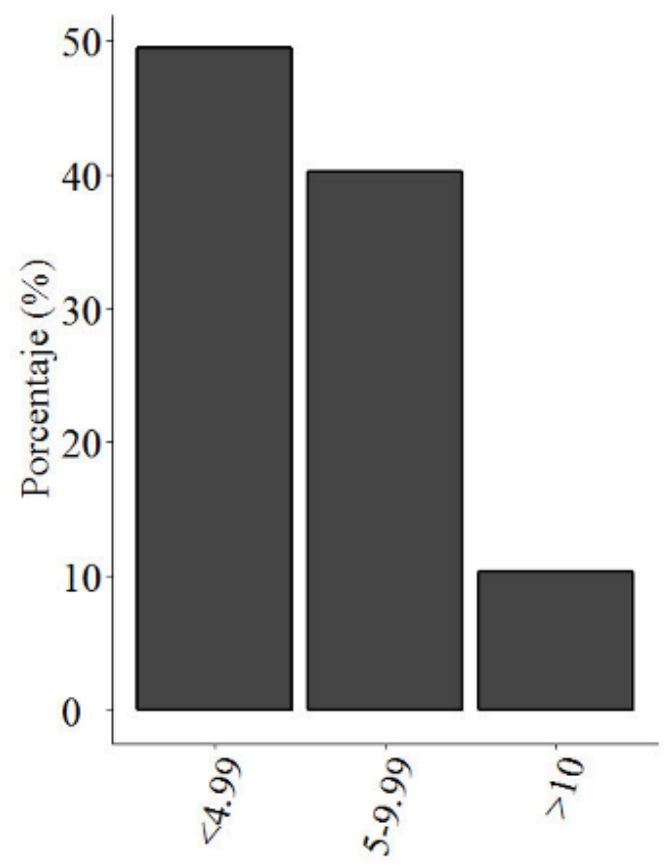

Clases altimétricas $(\mathrm{m})$

FIGURA 3. a) Distribución horizontal presentada en clases diamétricas para los tallos $\geq 3 \mathrm{~cm}$ DAP registrados en las parcelas muestreadas; b) Distribución vertical presentada en clases altimétricas para todos los individuos de árboles registrados en las parcelas muestreadas en el Valle de Agalta.

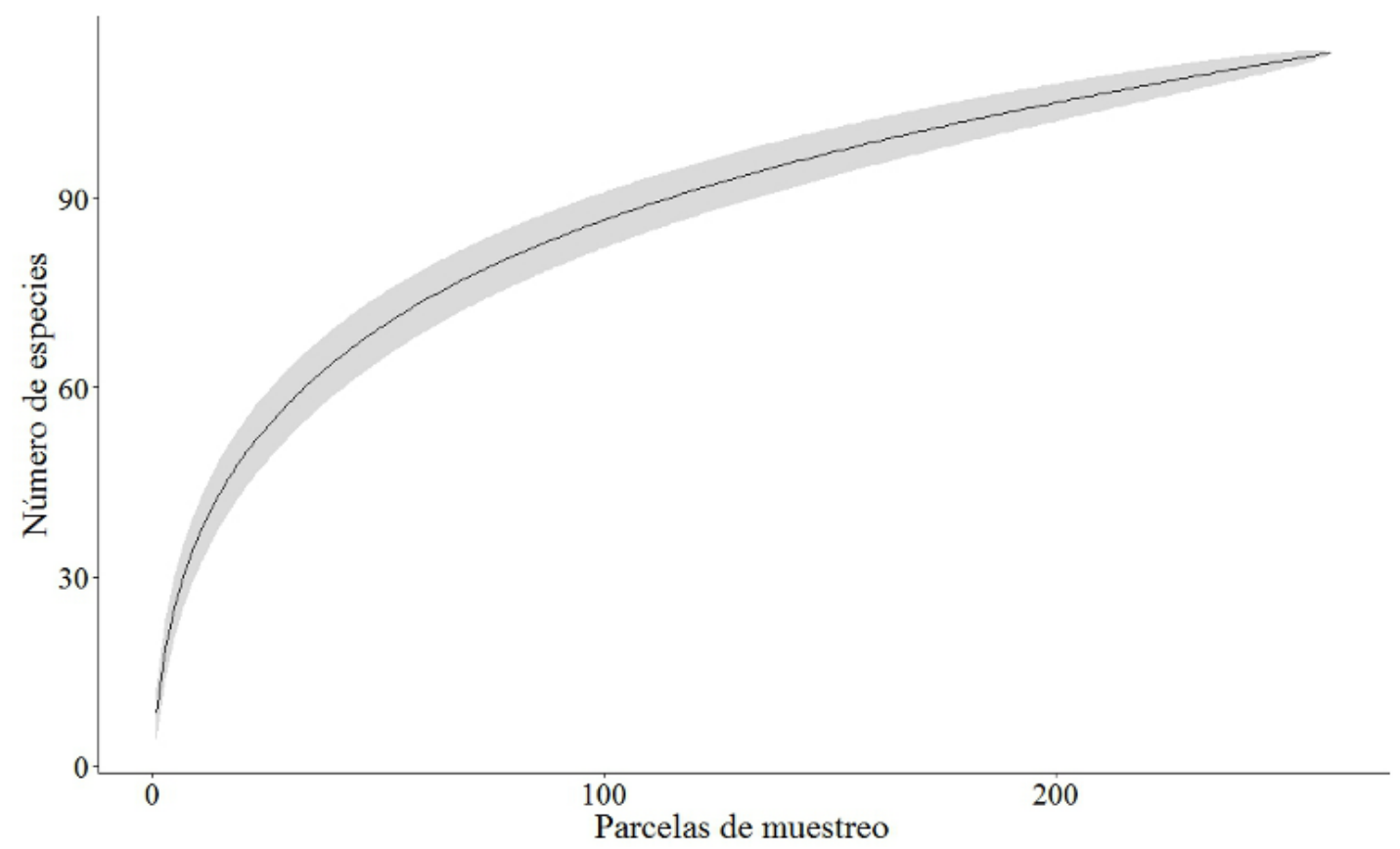

FIgURA 4. Curva de acumulación de especies en remanentes de BST en el Valle de Agalta. 


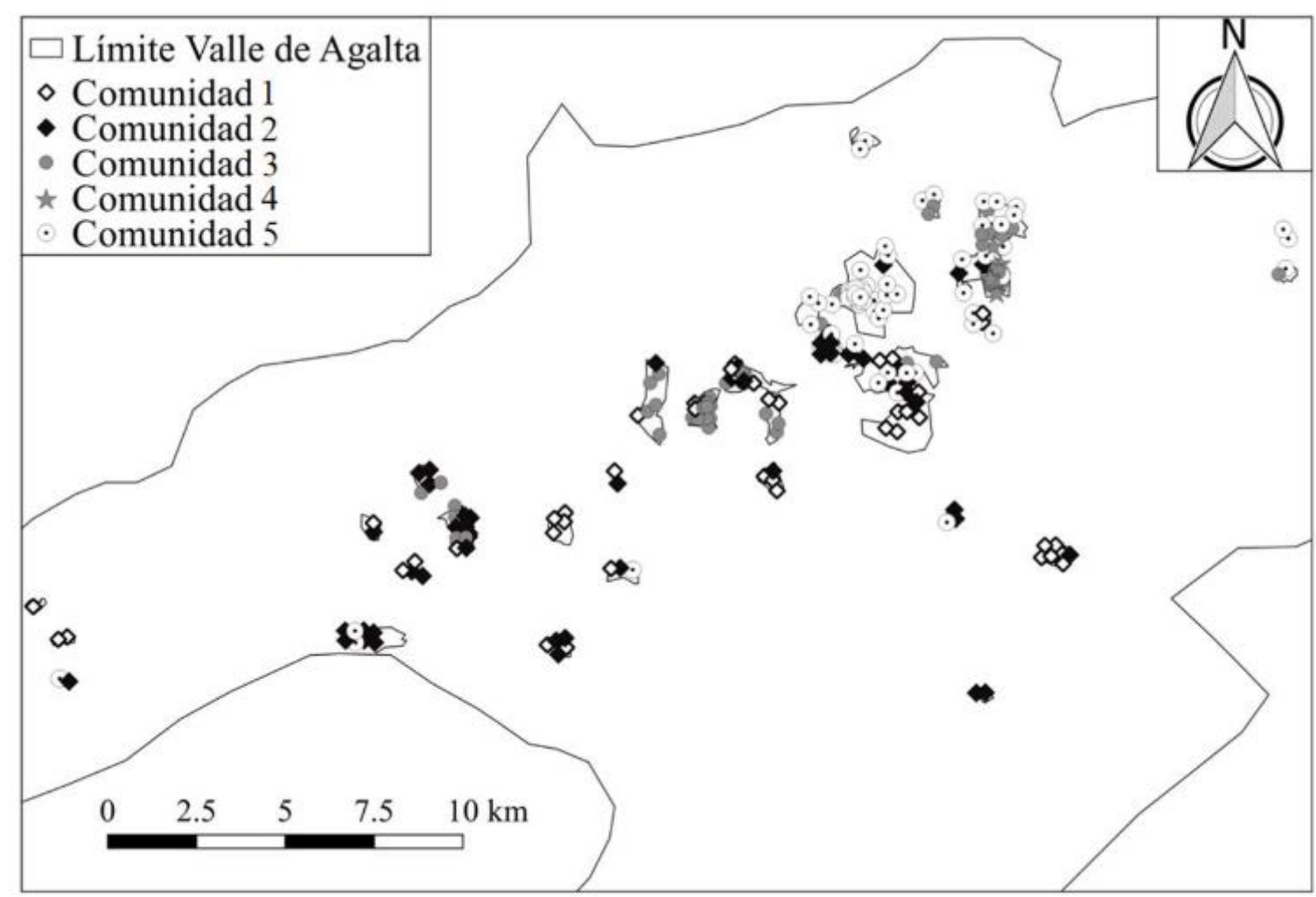

FIGURA 5. Distribución de comunidades distinguidas a través del análisis de aglomeración en el Valle de Agalta.

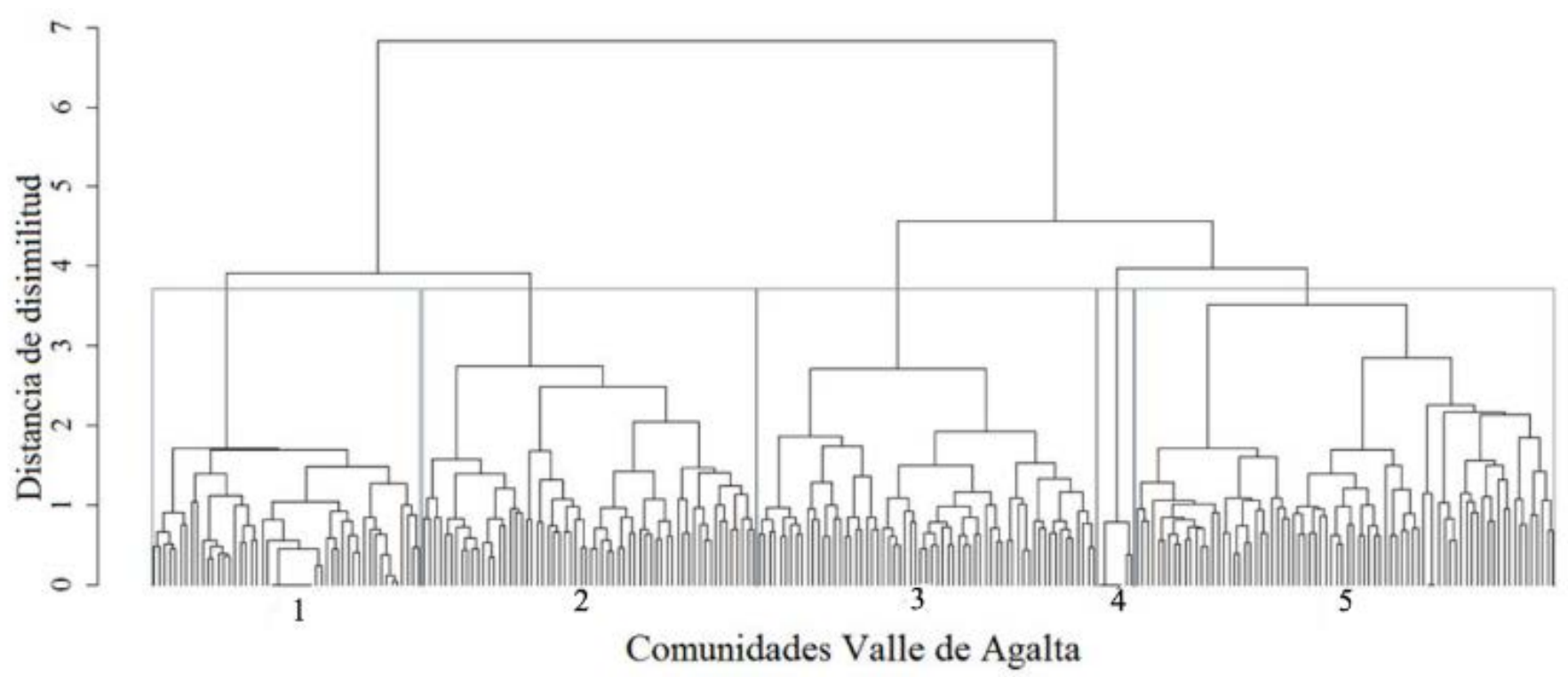

FigurA 6. Comunidades presentes en el Valle de Agalta, visualizadas en un dendrograma. 
TABLA 1. Parámetros de estructura vegetal de las parcelas de muestreo en el Valle de Agalta.

\begin{tabular}{lccccc}
\hline $\begin{array}{l}\text { Comunidades/P } \\
\text { arámetro }\end{array}$ & $\begin{array}{c}\text { Densidad } \\
\text { promedio (tallos } \\
\text { por hectárea) }\end{array}$ & $\begin{array}{c}\text { Altura } \\
\text { promedio }(\mathrm{m})\end{array}$ & $\begin{array}{c}\text { Densidad de } \\
\text { tallos }<3 \mathrm{~cm} \\
\text { DAP/ha }\end{array}$ & $\begin{array}{c}\text { Área basal } \\
\left(\mathrm{m}^{2} / \mathrm{ha}\right)\end{array}$ & $\begin{array}{c}\text { Cobertura } \\
\text { del dosel (\%) }\end{array}$ \\
\hline $\begin{array}{l}\text { Comunidad 1 } \\
\text { Árboles }\end{array}$ & $573.6 \pm 75.12$ & $7.42 \pm 0.32$ & $1,610.4 \pm 223.3$ & $1.2 \pm 0.12$ & $17.95 \pm 3$ \\
Arbustos & $6,395 \pm 1,161.6$ & $1 \pm 0.06$ & - & - & - \\
Cactáceas* $^{747.6 \pm 98.42}$ & $1.6 \pm 0.2$ & - & - & -
\end{tabular}

Comunidad 2

\begin{tabular}{|c|c|c|c|c|c|}
\hline Árboles & $966.8 \pm 55.85$ & $5.9 \pm 0.21$ & $2,206.4 \pm 203.6$ & $1.29 \pm 0.1$ & $28 \pm 3.1$ \\
\hline Arbustos & $7,840.7 \pm 753$ & $1 \pm 0.1$ & - & - & - \\
\hline Cactáceas* & $810.6 \pm 130.2$ & $1.9 \pm 0.1$ & - & - & - \\
\hline \multicolumn{6}{|c|}{ Comunidad 3} \\
\hline Árboles & $829.5 \pm 55.3$ & $5.2 \pm 0.2$ & $1,861.6 \pm 167.7$ & $0.9 \pm 0.1$ & $21.6 \pm 3.2$ \\
\hline Arbustos & $10,621 \pm 1,285.2$ & $1.1 \pm 0.1$ & - & - & - \\
\hline Cactáceas* & $508.9 \pm 60.4$ & $2.4 \pm 3.2$ & - & - & - \\
\hline \multicolumn{6}{|c|}{ Comunidad 4} \\
\hline Árboles & $122.9 \pm 47.1$ & $5.8 \pm 0.4$ & $131.4 \pm 59.7$ & $0.2 \pm 0.1$ & $2.3 \pm 1.5$ \\
\hline Arbustos & $17,625 \pm 5,334$ & $1 \pm 0.4$ & - & - & - \\
\hline Cactáceas* & $342.9 \pm 342.9$ & $0.1 \pm 0.1$ & - & - & - \\
\hline \multicolumn{6}{|c|}{ Comunidad 5} \\
\hline Árboles & $891.3 \pm 69.5$ & $5.8 \pm 0.2$ & $1,614.9 \pm 148.4$ & $0.7 \pm 0.1$ & $19.1 \pm 2$ \\
\hline Arbustos & $9,392.6 \pm 1,652.3$ & $1 \pm 0.04$ & - & - & - \\
\hline Cactáceas* & $257.2 \pm 32.9$ & $1.7 \pm 0.2$ & - & - & - \\
\hline
\end{tabular}

TABLA 2. Resumen de parámetros de estructura vegetal de las parcelas de muestreo para cobertura suelo.

\begin{tabular}{ccccc}
\hline $\begin{array}{c}\text { Comunidades/ } \\
\text { Parámetro }\end{array}$ & $\begin{array}{c}\text { Gramíneas } \\
\text { (\%) }\end{array}$ & $\begin{array}{c}\text { Hierbas } \\
(\%)\end{array}$ & $\begin{array}{c}\text { Suelo } \\
\text { desnudo } \\
(\%)\end{array}$ & $\begin{array}{c}\text { Hojarascas } \\
\text { (\%) }\end{array}$ \\
\hline 1 & $23.4 \pm 4.5$ & $41.1 \pm 6.2$ & $18.5 \pm 3.3$ & $12.5 \pm 2.7$ \\
2 & $10.7 \pm 3.3$ & $35.2 \pm 4$ & $17.8 \pm 2.8$ & $28.6 \pm 3.9$ \\
3 & $30.7 \pm 4.7$ & $25 \pm 3.3$ & $16.6 \pm 2.5$ & $23 \pm 4$ \\
4 & $87.7 \pm 8.4$ & $9.3 \pm 5.8$ & $2.6 \pm 2.6$ & $0.1 \pm 0.1$ \\
5 & $25.5 \pm 4$ & $13.9 \pm 2$ & $8.7 \pm 1.9$ & $40.6 \pm 4$ \\
\hline
\end{tabular}


TABLA 3. Coeficientes de variación (\%), de las variables estructurales registradas en cinco comunidades de BST del Valle de Agalta. Incluye número de parcelas (n) por cada comunidad.

\begin{tabular}{lccccc}
\hline Variable & \multicolumn{5}{c}{ Comunidad } \\
& $\begin{array}{c}1 \\
(n=50)\end{array}$ & $\begin{array}{c}2 \\
(n=62)\end{array}$ & $\begin{array}{c}3 \\
(n=63)\end{array}$ & $\begin{array}{c}4 \\
(n=7)\end{array}$ & $\begin{array}{c}5 \\
(n=78)\end{array}$ \\
\hline DAP & 73.6 & 32.4 & 26.9 & 42.3 & 86.5 \\
Altura árboles & 31 & 27.6 & 24.2 & 20.5 & 32.8 \\
Altura arbustos & 45.6 & 42.6 & 46 & 39.7 & 40.3 \\
Altura cactáceas & 83.3 & 46.4 & 69.9 & 264.6 & 87.5 \\
Densidad de arbustos & 128.4 & 75.6 & 96 & 80.1 & 155.4 \\
Densidad de cactáceas & 93.1 & 126.5 & 94.1 & 264.6 & 112.9 \\
Densidad de árboles & 92.6 & 45.6 & 53 & 101.3 & 68.9 \\
\hline
\end{tabular}

Comunidad 2: Esta comunidad ( $\mathrm{n}=62$ parcelas, Figura $7 \mathrm{~b}$ ) se caracterizó por poseer alta abundancia relativa de $A$. picachensis $(24.3 \%$ ) y el mayor índice de valor de importancia (25.6\%). Sin embargo, la distribución de abundancia relativa de las plantas leñosas fue más equitativa en comparación con la comunidad 1. Las especies que acompañaron a $A$. picachensis con los valores más altos de abundancia relativa (intervalo $=2.6 \%-13.4 \%$ ) fueron Acacia pennatula, $B$. simaruba, L. leucocephala, Zanthoxylum fagara, R. aculeata, Phyllostylon rhamnoides, $N$. paniculata y Casearia nitida.

Comunidad 3: Esta comunidad ( $\mathrm{n}=63$ parcelas, Figura 7c) consistió de las siguientes especies de plantas leñosas: E. areolatum (15.3\%), A. picachensis (13.03\%), C. nitida (12\%), Z. fagara $(10.3 \%)$, C. acapulcense (9.6\%), B. simaruba (5.2\%), Eugenia hondurensis (5.1\%), Lysiloma acapulcense (3.3\%), $A$. collinsi (2.8\%), Schoepfia schreberi (2.7\%), T. calderoni (2.4\%), Randia cookii (2.4\%), y Megastigma skinneri (2\%). En esta comunidad, E. areolatum (15.3\%) obtuvo el IVI más alto, seguida por $A$. picachensis (14.3\%) y C. acapulcensis (9.5\%).

Comunidad 4: Esta comunidad ( $\mathrm{n}=7$ parcelas, Figura $7 \mathrm{~d}$ ) se caracterizó por poseer la abundancia relativa y valor de importancia más alto de Lysiloma acapulcense con 93.1\% y
$86.1 \%$, respectivamente. Esta especie estuvo acompañada por Acacia pennatula con 4.9\% y Acacia collinsi con 2\%. L. acapulcense estuvo presente en 2 comunidades más (comunidad 3 y 5 con abundancias relativas de $3.3 \%$ y $0.3 \%$, respectivamente). Esta comunidad tuvo una cualidad de estructura más abierta

Comunidad 5: Esta comunidad ( $\mathrm{n}=78$ parcelas, Fig. $7 \mathrm{e}$ ) se caracterizó por poseer una alta abundancia relativa (19.15\%) de Eugenia hondurensis; especie con el mayor índice de valor de importancia (17.2\%). Las otras especies que acompañaron a E. hondurensis con intervalos de abundancia relativa entre $2 \%$ y $12.14 \%$ fueron Acer negundo, Nectandra coriacea, Z. fagara, E. areolatum, Acacia farnesiana, Eugenia acapulcensis, C. acapulcensis, A. collinsi, Cochlospermum vitifolium, C. nitida, B. simaruba, Luehea candida y Triumfetta calderoni.

\section{Riqueza de especies, diversidad y complejidad de sitios}

La comunidad 5 tuvo la mayor riqueza de especies de plantas leñosas y la comunidad 3 obtuvo la menor riqueza de especies, a la vez siendo el sitio con menor complejidad estructural (Tabla 3. 

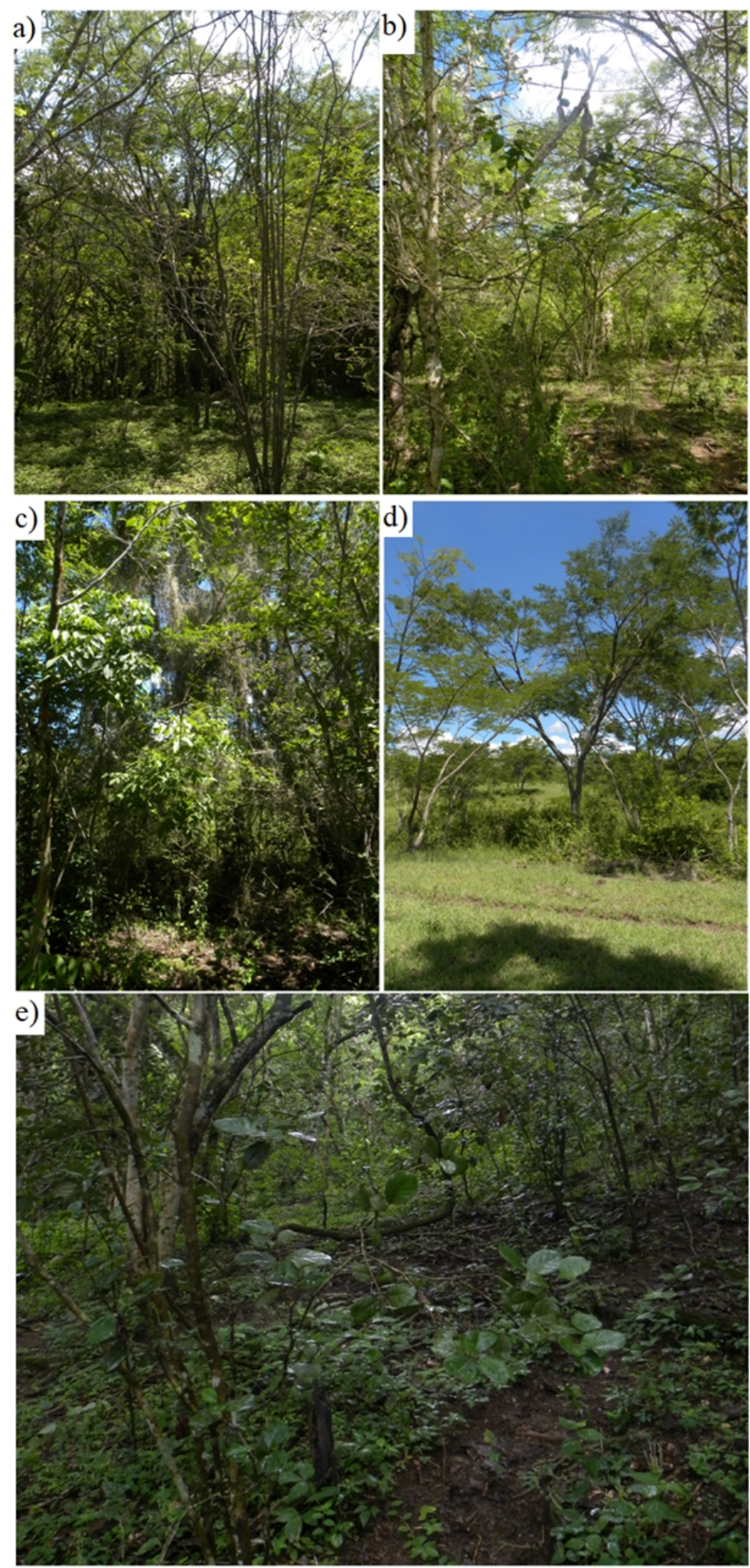

FIGURA 7. Comunidades vegetales del BST del Valle de Agalta: a) Comunidad 1 en el sector de Pie de Anona, municipalidad de San Esteban; b) Comunidad 2 en el sector de Potrero de Casas, municipalidad de Gualaco; c) Comunidad 3 en el sector de El Barro, San Esteban; d) Comunidad 4 en el sector de El Barro, San Esteban y e) Comunidad 5 en el sector de Potrerillos, San Esteban.

\section{Especies de importancia para la conservación}

En el Valle de Agalta se encuentran especies de orquídeas registradas en el Apéndice II de CITES (2016): Sarcoglottis sceptrodes, Oncidium cebolleta, Myrmercophila wendlandii, Epidendrum rigidum, Brassavola appendiculata, Encyclia nematocaulon, Barkeria obovata, Habenaria novemfida, Prosthechea chacaoensis, Spiranthes polyantha, Ionopsis utricularioides, y Maxillaria variabilis.

El estado de las especies según la lista Roja de UICN: gravemente amenazada: Casearia williamsiana; amenazada se enlistan: Guaiacum sanctum, Peniocereus chiapensis, Xolocotzia asperifolia; de preocupación menor: Tillandsia fasciculata, Rhipsalis baccifera, Acanthocereus tetragonus, Hylocereus minutiflorus, Chamaecrista nictitans, Hymenaea courbaril, Calliandra formosa, Mimosa albida, Dalea scandens, Ludwigia octovalvis, Eragrostis hondurensis, Chiococca alba, Casearia aculeata; y vulnerable: Phyllostylon orthopterum.

El BST del Valle de Agalta posee especies endémicas y raras. Estas especies no se encuentran distribuidas de manera homogénea. En el sector de Palo Verde se registra Robinsonella erasmi-sosae, y Eugenia hondurensis. En Maguelar se registra Bakeridesia molinae, E. hondurensis, Chiococca alba y Xolocotzia asperifolia. En Potrerillos hay presencia de R. erasmi-sosae, E. hondurensis, y Bernardia nicaraguensis. Mientras que en San Miguel se encontró Bernardia nicaraguensis, Calliandra formosa, E. hondurensis, Rondeletia hondurensis, y Casearia williamsiana. En el Barro se registra Bernardia nicaraguensis, Aphananthe monoica, Casearia williamsiana y E. hondurensis.

\section{DISCUSIÓN}

\section{Patrones de comunidades florísticas}

La composición florística del Valle de Agalta fue muy diversa y poco abundante. Las distribuciones diamétrica y altimétrica indican que el bosque está formado por individuos jóvenes. Estas distribuciones han sido observadas en otros bosques secos, Aguirre Mendoza et al. (2014) mencionan que este tipo de distribución se debe a que estos bosques han sido sometidos a la extracción de 
madera y cambios de uso de suelo. El Valle de Agalta, durante años ha sido un área muy importante para la ganadería y la agricultura en el país, lo que ha conllevado a la fragmentación del bosque. Por estas cualidades, se concluye que la mayor parte del bosque se califica como bosque en sucesión temprana, que se caracteriza por un proceso dinámico de cambios. Los impactos de las transformaciones antrópicas como la ganadería y la agricultura traen como consecuencias una reducción de la biodiversidad, de la cobertura vegetal del suelo, alteración del ciclo del agua, y cambios en los nutrientes del suelo (García-Oliva y Jaramillo, 2010), así como en la polinización y sistemas de reproducción en especies vegetales de este ecosistema (Quesada et al., 2010).

El ecosistema del Valle de Agalta está compuesto por especies caducifolias como Fabaceae, así como de familias perennifolias como Rubiaceae, Capparaceae, entre otras. Estudios sobre composición florística han registrado la familia Fabaceae como la más común en algunos ecosistemas, entre ellos: Sánchez-Rodríguez et al. (2003) en el bosque mesófilo de Montaña en la Sierra de Manantlán, Jalisco en México. En los Asentamientos Campesinos "Las Delicias" y "El Guamo" en el Estado de Bolivar, Venezuela (Díaz, 2007); en tierras bajas de Madidi en Bolivia (Quisbert y Macía, 2005); la Estación Biológica Caparú, Vaupés en la Amazonia Colombiana (Cano y Stevenson, 2009), y en Zapotillo, provincia de Loja en Ecuador (AguirreMendoza, Buri Sivisaca, Betancourt y Geada López, 2014).

El Valle de Agalta posee los once géneros que representan las seis regiones fitogeográficas de BST estudiadas por Gentry (1995), siendo: Tabebuia, Cordia, Casearia, Erytroxylum, Baubinia, Trichilia, Randia, Hippocratea, Serjania, Croton, Zanthoxylum. La flora de este Valle está constituida por especies nativas, con presencia de pocas especies introducidas. La introducción de estas especies exóticas se debe a las actividades de reforestación entre estas: Leucaena leucocephala, y Moringa oleifera; para cultivos agrícolas: Cucumis anguria, Gossipium birsutum y otras plantas introducidas para pasto como Rhynchelytrum repens, y Portulaca oleracea, así como invasoras, Oeceoclades maculata. Además, este estudio enriquece el conocimiento de la distribución de las plantas, en particular aquellas de interés por su estado de conservación. Por ejemplo, Bakeridesia molinae se registró de elevaciones entre $481 \mathrm{~m}$ y 593 m snm, representó una distribución altitudinal distinta a la de su ejemplar tipo $1800 \mathrm{~m}$ snm (Sutherland, 2001). Robinsonella erasmi-sosae se registró a 499 m y 532 m snm y su holotipo fue registrado a $1500 \mathrm{~m}$ snm. (Sutherland, 2001).

El cálculo de la riqueza de especies sugiere que aún existen especies sin registrar en los remanentes de BST del Valle de Agalta. Kalacska et al. (2004) indicaron que la presencia de especies raras influyó en los estimados de riqueza de especie total en un BST de Costa Rica. En comparación en el Valle de Agalta se registró 42 especies con abundancias absolutas de 1-5 individuos. Estos resultados sugieren que muestreos como los realizados en este estudio, son necesarios para lograr captar especies raras y de interés para la conservación.

Considerando lo anterior, las asociaciones vegetales identificadas se asemejan a otras presentes en bosques secos del continente. Las asociaciones vegetales con IVI elevado de Acacia picachensis (1 y 2) son semejantes a la asociación vegetal denominada bosque espinoso (Leopold, 1950; Rzedowski, 2006) y la comunidad con IVI elevado de Eugenia hondurensis (5) es comparables con el bosque deciduo latifoliado (Programa Reducción de las emisiones derivadas de la deforestación y la degradación de los bosques $[\mathrm{REDD}+]$ Comisión Centroamericana de Ambiente y Desarrollo [CCAD]/ Deutsche Gesellschaft für Internationale Zusammenarbeit [GIZ], 2011) y en parte con el bosque deciduo bajo (Mora et al., 2015). La asociación vegetal 4 parece ser el resultado de un proceso selectivo en el cual no se talaron todos los individuos de Lysiloma acapulcensis. Todas las parcelas de esta asociación se encuentran en un sector del Valle (San Miguel/El Barro). 
Importancia de Valle de Agalta para la conservación del BST

Se ha concluido que los bosques secos de Latino América son importantes por su alta diversidad, riqueza y recambio de especies (Banda et al., 2016). En Honduras el estado de protección de los bosques secos es casi nulo. De estos esfuerzos de conservación destacan la designación del Sitio de Importancia para la Vida Silvestre El Ciruelo en el Valle de Agalta en el departamento de Olancho (Instituto Nacional de Conservación y Desarrollo Forestal, Áreas Protegidas y Vida Silvestre [ICF], 2015) y el Refugio de Vida Silvestre Colibrí Esmeralda Hondureño en el Valle de Aguán departamento de Yoro ((Instituto Nacional de Conservación y Desarrollo Forestal, Áreas Protegidas y Vida Silvestre [ICF], 2011). Sin embargo, estos esfuerzos apenas suman 2,079 hectáreas y el BST en esta región se ve amenazado por la pérdida de cobertura generada por el cambio de uso de suelo (Miles et al., 2006). El Valle de Agalta aún posee BST que representa un hábitat único y es el refugio de muchas especies nativas, raras y endémicas, enlistándose algunas en estado amenazado y vulnerable; por esta razón se considera un hábitat de prioridad alta de conservación.

\section{CONCLUSIONES}

Los bosques secos se consideran uno de los ecosistemas con mayor amenaza en el trópico debido a las actividades antrópicos como la ganadería y la agricultura, en particular en Honduras donde se han declarado muy pocas áreas protegidas que abarcan este ecosistema.

La mayoría de las especies registradas son nativas y algunas son gramíneas y fabáceas introducidas debido a la ganadería. Este estudio contribuyó a conocer el hábitat del colibrí esmeralda, Amarilia luciae, una especie endémica que habita en los bosques secos de Honduras, y es de gran aporte para estudios futuros como la dinámica del ecosistema. Con base en esta investigación, también se identificará la fragmentación en las áreas de estudio representativas del BST en Honduras y, de esta manera, se podrán proponer corredores que permitan la conectividad ecológica entre fragmentos. Esto podría asegurar el flujo génico entre las poblaciones.

\section{RECONOCIMIENTOS}

Se agradece al programa "Corredor Agrícola" financiado por el Banco Interamericano de Desarrollo [BID], ejecutado por la entidad gubernamental de Honduras, Inversión Estratégica Honduras [INVESTHHonduras]/Cuenta Desafío del Milenio- Honduras [MCAH]. A los biólogos Rosa Ramírez y Denis Padilla por su colaboración en el trabajo de campo y al Herbario TEFH por facilitar a los autores el uso de la colección general y de las instalaciones para el secado, identificación y montaje del material. También agradecemos la colaboración de los especialistas por la identificación de especies y así como a los revisores anónimos. Finalmente, se agradece a la Asociación de Investigación para el Desarrollo Económico y Socioecológico (ASIDE) y American Bird Conservancy por su acompañamiento durante el proyecto.

\section{REFERENCIAS}

Aguirre-Mendoza, Z., Buri Sivisaca, D., Betancourt, Y., \& Geada López, G. (1994). Composición florística, estructura y endemismo en una parcela permanente de bosque seco en Zapotillo, provincia de Loja, Ecuador. Arnaldoa, 21(1), 165178. doi: $10.13140 / 2.1 .1288 .0008$

Austin, M. P., \& Greig-Smith, P. (1968). The application of quantitative methods to vegetation survey: II. Some methodological problems of data from rainforest. Journal of Ecology, 56(3), 827-844. doi: 10.2307/2257889

Banda, K, Delgado-Salinas A., Dexter, K.G., Linares-Palomino, R., Oliveira-Filho, A., Prado, D., ... , \& Pennington, R.T. (2016). Plant diversity patterns in neotropical dry forests and their conservation implications. Science, 353(6306), 13831387. doi: 10.1126/science.aaf5080

Barrance, A., Schreckenberg, K., \& Gordon, J. (2009). Conservación mediante el uso: Lecciones aprendidas en el bosque seco tropical mesoamericano. London: Overseas Development Institute.

Bibby, C. J., Burgess, N. D., Hill, D. A., \& Mustoe, S. (1992). Bird Census Techniques. London: Academic Press.

Burgman, M. A. \& Thompson, E. J. (1982). Cluster analysis, ordination and dominance-structural classification applied to diverse tropical vegetation at Jabiluka, Northern 
Territory. Australian Journal of Ecology, 7(4), 375-387. doi: 10.1111/j.1442-9993.1982.tb01312.x

Cano, A., \& Stevenson, P. R. (2009). Diversidad y composición florística de tres tipos de bosque en la Estación Biológica Caparú, Vaupés. Revista Colombia Forestal, 12(1), 63-80. doi: 10.14483/udistrital.jour.colomb.for.2009.1.a06

Carbajal-Vásquez, J. S. (2010). Vegetación del "Corredor Biológico de Uso Múltiple Texiguat”, El Paraíso, Honduras. Tesis de licenciatura, Escuela Agrícola Panamericana, San Antonio de Oriente, Francisco Morazán, Honduras.

Cardona, R. A. \& Oramas, S.A. (2002). Composición florística de la Selva Baja Caducifolia en Quebrada Grande, Morocelí, El Paraíso. Tesis de licenciatura, Escuela Agrícola Panamericana, San Antonio de Oriente, Francisco Morazán, Honduras.

Chiarucci, A. (2001). Evaluation and monitoring of the flora in a nature reserve by estimation methods. Biological Conservation, 101(3), 305-314. doi: 1016/S0006-3207(01)00073-8

Centro Internacional de Agricultura Tropical [CIAT] (1999a). Lluvia anual, temperatura minima, temperatura máxima, elevación $(5 \mathrm{~km})$ de Honduras. Recuperado de http//gisweb.ciat.cgiar.org [Cited: Sept 15 2016].

Centro Internacional de Agricultura Tropical [CIAT] (1999b). Suelos de Honduras clasificación FAO. Recuperado de http//gisweb.ciat.cgiar.org. [Cited: Sept 15 2016].

Convención sobre el Comercio Internacional de Especies Amenazadas de Fauna y Flora Silvestres [CITES]. Appendices I, II and III. Recuperado de http// cites.org: 2018

Curtis, J. T., \& McIntosh, R. P. (1951). An upland forest continuum in the prairie-forest border region of Wisconsin. Ecology, 32(3), 476-496. doi.org/10.2307/1931725

Díaz, P.W.A. (2007). Composición florística y estructura de bosques en los asentamientos campesinos Las Delicias, el Guamo y Lechozal, Estado Bolívar, Venezuela. Ernstia, 17(1), 1-24.

Duery-Salek, S. (2001). Caracterización del bosque seco de la comunidad de Oropoli, Honduras. Tesis de licenciatura, Escuela Agrícola Panamericana, San Antonio de Oriente, Francisco Morazán, Honduras.

Espinosa, C. I., de la Cruz, M., Luzuriaga, A. L., \& Escudero, A. (2012). Bosques tropicales secos de la región Pacífico Ecuatorial: diversidad, estructura, funcionamiento e implicaciones para la conservación. Ecosistemas, 21(1-2), 167-179. Recuperado de https://www.revistaecosistemas.net/index.php/ecosistema s/article/view/35
Ferrufino-Acosta, L., Díaz, R F., Benítez-Nassar, D., Atao, F. R., García-Chaclan, M., López, T., \& Pilz, G. E. (2018a). Composición florística de la finca agroecológica de Zamorano, Honduras: una experiencia del Programa Aprender Haciendo. Ceiba, 55(1), 1-20. doi:http://dx.doi.org/10.5377/ ceiba.v55i1.4680.

Ferrufino-Acosta, L., Sandoval, G., \& Oyuela, O. (2018b). Flora del Parque Arqueológico de Copán, Honduras. Revista Ciencia y Tecnología, 21(1), 71-89. doi: 10.5377/rct.v0i20.5923

Finegan, B. \& Bouroncle, C. (2007). Patrones de fragmentación de los bosques de tierras bajas, su impacto en las comunidades y especies vegetales y propuestas para su mitigación. En A. C. Harvey, \& J. C. Sáenz (Eds.). Evaluación y conservación de biodiversidad en paisajes fragmentados de Mesoamérica (pp. 139-178). Heredia: Instituto Nacional de Biodiversidad (INBio).

Garcés, P. A. (2004). Composición florística del bosque seco "Masicarán", Valle del Yeguare, Honduras, C.A. Zamorano. Tesis de licenciatura, Escuela Agrícola Panamericana, San Antonio de Oriente, Francisco Morazán, Honduras.

García-Oliva, F. \& Jaramillo, V. J. (2010). Impact of anthropogenic transformation of seasonally dry tropical forests on ecosystem biogeochemical processes. En R. Dirzo, H.S. Young, H.A. Mooney, \& G. Ceballos (Eds.). Seasonally dry tropical forest. Ecology and conservation (1 ${ }^{\mathrm{a}}$ ed.) (pp. 159-172). Washington, USA: Island Press /Center for Resource Economics. doi: 10.5822/978-1-61091-021-7.

García-Villacorta, R. (2009). Diversidad, composición y estructura de un hábitat altamente amenazado: Los bosques estacionalmente secos de Tarapoto, Perú. Revista Peruana de Biología, 16(1), 81-92. doi: 10.15381/rpb.v16i1.177

Gentry, A. (1995). Diversity and floristic composition of neotropical dry forests. En S. H. Bullock, H. A.Mooney, \& E. Medina (Eds.). Seasonally dry tropical forests (pp. 146-190). Cambridge: Cambridge University Press.

Gotelli, N. \& Colwell, R. (2011). Estimating species richness. En A. Magurran, \& B. J. McGill (Eds.). Biological diversity: frontiers in measurement and assessment (pp. 39-54). New York: Oxford University Press. doi:10.2307/3547060

Gutiérrez, J. F., \& Linares, G. M. (2002). Composición florística de la vegetación riparia de "Quebrada Grande". Moroceli, El Paraíso, Honduras, C.A. Zamorano. Tesis de licenciatura, Escuela Agrícola Panamericana, San Antonio de Oriente, Francisco Morazán, Honduras.

Hill, M. O. (1973). Diversity and evenness: A unifying notation and its consequences. Ecology, 54(2), 427-432. doi: $10.2307 / 1934352$ 
Holdridge, L. R. (1962). Mapa de las zonas de vida de Honduras, [mapa]. Escala 1:50,000.

Holdridge, L. R. (1967). Life Zone ecology. San Jose: Tropical Science Center.

House, P. R. (2004). El polígono refugio del Colibrí Esmeralda bondureño. Tegucigalpa: Unidad SIG Monitoreo Ecológico. Tegucigalpa, Honduras: Universidad Católica de Honduras.

Instituto Nacional de Conservación y Desarrollo Forestal, Áreas Protegidas y Vida Silvestre-[ICF] (2011). Decreto 204-2011. Comayagüela: Instituto Nacional de Conservación y Desarrollo Forestal, Áreas Protegidas y Vida Silvestre.

Instituto Nacional de Conservación y Desarrollo Forestal, Áreas Protegidas y Vida Silvestre [ICF] (2015). Acuerdo Número 005-2015. Comayagüela: Instituto Nacional de Conservación y Desarrollo Forestal, Áreas Protegidas y Vida Silvestre.

Instituto Nacional de Conservación y Desarrollo Forestal, Áreas Protegidas y Vida Silvestre [ICF] (2014). Mapa forestal y cobertura de la Tierra. Tegucigalpa: Unidad de Monitoreo Forestal Instituto Nacional de Conservación y Desarrollo Forestal, Áreas Protegidas y Vida Silvestre.

Illescas-Burneo, W. (2005). Caracterización florística del bosque seco Masicaran, Tatumbla, Honduras. C. A. Zamorano. Tesis de licenciatura, Escuela Agrícola Panamericana, San Antonio de Oriente, Francisco Morazán, Honduras.

James, F. C., \& Shugart, H. H. (1970). A quantitative method of habitat description. Audubon Field Notes, 24(6), 727-736. doi: $10.2307 / 1934352$

Janzen, D. H. (1988). Tropical dry forests the most endangered tropical ecosystem. En E. O. Wilson (Ed.). Biodiversity (pp.130-137). Washington D. C.: National Academy Press.

Kalacska, M., Sánchez-Azofeifa, G. A., Calvo-Alvarado, J. C., Quesada, M., Rivard, B., \& Janzen, D. H. (2004). Species composition, similarity and diversity in three successional stages of a seasonally dry tropical forest. Forest Ecology and Management, 200(1), 227-247. doi: 10.1016/j.foreco.2004.07.001

Leopold, A. S. (1950). Vegetation zones of Mexico. Ecology, 31(4), 507-518. doi: 10.2307/1931569

Lesko, C. P. (2005). Composición florística estructural del bosque de galería de la Quebrada Grande, Morocelí, El Paraíso, Honduras, C. A. Zamorano. Tesis de licenciatura, Escuela Agrícola Panamericana, San Antonio de Oriente, Francisco Morazán, Honduras.

Martin, T. E., Paine, C., Conway, C. J., Hochachka, W. M., Allen, P., \& Jenkins, W. (1997). Breeding biology research and monitoring database (BBIRD) field protocol. Missoula: Montana Cooperative Wildlife Unit.

Menéndez-Posada, R.N., \& Melara-Estrada, N.W. (2002). Composición florística del bosque de galería de la Quebrada Güisisire, Moroceli, El Paraíso, Honduras, C.A. Tesis de licenciatura, Escuela Agrícola Panamericana, San Antonio de Oriente, Francisco Morazán, Honduras.

Midence, C. M. (2016). Áreas complementarias para la conservación de la flora de preocupación especial en regiones semiáridas de Honduras. Ceiba, 54(2), 147-156. di: $10.5377 /$ ceiba.v54i2.3285

Miles, L., Newton, A. C., Defries, R., Ravilious, C., May, I., Blyth, S., Kapos, V., \& Gordon, J.E. (2006). A global overview of the conservation status of tropical dry forests. Journal of Biogeography, 33(3), 491-505. doi: 10.1111/j.13652699.2005.01424.x

Mora, J. M., Espinal, M., López, L. I., \& Quezada, B. O. (2015). Caracterización del bosque seco tropical remanente en el Valle de Agalta, Honduras. Ceiba, 53(1), 38-56. doi: 10.5377/ceiba.v53i1.2015

Murtagh, F., \& Legendre, P. (2014). Ward's hierarchical agglomerative clustering method: Which algorithms implement Ward's criterion?. Journal of Classification, 31(3), 274-295. doi: 10.1007/s00357-014-9161-z

NASA Land Processes Distributed Active Archive Center [LP DAAC] (2001). ASTER Global DEM V2. Recuperado de: http//gdex.cr.usgs.gov.

Nassar, J. M., Rodríguez, J. P., Sánchez-Azofeifa, A., Garvin, T., \& Quesada, M. (2008). Human, ecological and biophysical dimensions of Tropical Dry Forests. Caracas: Instituto Venezolano de Investigaciones Científicas.

Oksanen, J., Blanchet, F. G., Kindt, R., Legendre, P., Minchin, P. R., O’hara, R. B., Simpson, G. L., Solymos, P., Stevens, M. H. H., \& Wagner, W. (2017). vegan: Community Ecology Package. R Package version 2.4-2.

Orloci, L. (1967). An agglomerative method for classification of plant communities. Journal of Ecology, 55(1), 193-206. doi: $10.2307 / 2257725$

Pennington, R. T, Lewis, G. P., \& Ratter, J. A. (2006). Neotropical savannas and seasonally dry forests: plant diversity, biogeography and conservation. Boca Raton: Taylor \& Francis.

Pérez-Laniado, M. E. (2004). Composición florística del Cerro Masicarán, San Antonio de Oriente, Honduras. Tesis de licenciatura, Escuela Agrícola Panamericana, San Antonio de Oriente, Francisco Morazán, Honduras. 
Programa Reducción de las emisiones derivadas de la deforestación y la degradación de los bosques [REDD+] Comisión Centroamericana de Ambiente y Desarrollo [CCAD]/ Deutsche Gesellschaft für Internationale Zusammenarbeit [GIZ] (2011). Tipos de bosques y contexto del mapeo de la cobertura forestal en Centroamérica y República Dominicana. Antigua. Deutsche Gesellsachaft für Internationale Zusammenarbeit.

Quesada, M., Rosas, F., Aguilar, R., Ashworth, L., RosasGuerrero, V. M., Sayago, R., Lobo, J. A., Herrerías-Diego, Y., \& Sánchez-Montoya, G. (2010). Human impacts on pollination, reproudction, and breeding systems in tropical forest plants. En R. Dirzo, H. S. Young, H. A. Mooney, \& G. Ceballos (Eds.). Seasonally dry tropical forest. Ecology and conservation (1 ${ }^{\mathrm{a}}$ ed.) (pp. 173-194). Washington, USA: Island Press /Center for Resource Economics. doi: 10.5822/978-1-61091-021-7

Quisbert, J., \& Macía, M. J. (2005). Estudio comparativo de la composición florística y estructura del bosque de tierra firme en dos sitios de tierras bajas de Madidi. Ecología en Bolivia, 40(3), 339-364.

R Core team. R. (2016). A language and environment for statistical computing. Vienna: R Foundation for Statistical Computing.

Rao, C. (1995). A review of canonical coordinates and an alternative to correspondence analysis using hellinger distance. Questiió, 19(1), 22-63. Recuperado de http://hdl.handle.net/2099/4059

Ruíz, L. J., \& Fandiño, M. C. O. (2009). Estado del bosque seco tropical e importancia relativa de su flora leñosa, Islas de la Vieja Providencia y Santa Catalina, Colombia, Caribe Suroccidental. Revista de la Academia Colombiana de Ciencias Exactas, Físicas y Naturales, 33(126), 7-15. Recuperado de http://www.accefyn.com/revista/Vol_33/126/5-16.pdf

Rzedowski, J. (2006). Vegetación de México. México D. F.: Comisión Nacional para el Conocimiento y Uso de la Biodiversidad.
Sánchez-Rodríguez, E. V., López-Mata, L., García-Moya, E., \& Guzmán, R. C. (2003). Estructura, composición florística y diversidad de especies leñosas de un bosque mesófilo de montaña en la Sierra de Manantlán, Jalisco. Botanical Sciences, 73(1), 17-34. doi: 10.17129/botsci.1676

Secretaría de Energía, Recursos Naturales, Ambiente y Minas (2014). V Informe Nacional de Biodiversidad. Tegucigalpa: $\mathrm{CBD} / \mathrm{GEF} / \mathrm{PNUD}$.

Sutherland, C. N. (2001). Plantas descritas originalmente de Honduras y sus nomenclaturas equivalentes actuales. Ceiba, 42(1), 1-71. Recuperado de https://revistas.zamorano.edu/index.php/CEIBA/article/ view/409

Walther, B. A., \& Moore, J. L. (2005). The concept of bias, precision and accuracy, and their use in testing the performance of species richness estimators, with a literature review of estimators. Ecography, 28(6), 815-829. doi: 10.1111/j.2005.0906-7590.04112.x

Ward, J. H. (1963). Hierarchical grouping to optimize an objective function. Journal of the American Statistical Association, 58(301), 236-244. doi: 10.1080/01621459.1963.1050084

Manuscrito recibido el 25 de septiembre de 2017

Aceptado el 11 de diciembre de 2018

Publicado el 16 de octubre de 2019

Este documento se debe citar como:

Ferrufino-Acosta, L., Cruz, S. Y., Mejía O, T., Rodríguez, F., Escoto, D., Sarmiento, E., \& Larkin, J. L. (2019). Composición, estructura y diversidad florística del bosque seco en el Valle de Agalta, Honduras. Madera y Bosques, 25(2), e2521635. doi: 10.21829/myb.2019.2521635

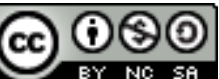

Madera y Bosques por Instituto de Ecología, A.C. se distribuye bajo una Licencia Creative Commons Atribución-NoComercialCompartirlgual 4.0 Internacional. 